\title{
Polyacrylamide-Coated Capillaries Probed by Atomic Force Microscopy: Correlation between Surface Topography and Electrophoretic Performance
}

\author{
Alejandro Cifuentes and J ose C. Díez-Masa* \\ Institute of Organic Chemistry (CSIC), Juan de la Cierva 3, 28006 Madrid, Spain \\ J Urgen Fritz and Dario Anselmetti \\ Novartis Services AG, Scientific Services, Physics, CH-4002 Basel, Switzerland
}

\author{
Alfredo E. Bruno* \\ Novartis Pharma AG, Bioanalytics \& Pharmacokinetics, CH-4002 Basel, Switzerland
}

The inner wall surface topography of fused silica capillaries with an inner diameter of $50 \mu \mathrm{m}$, coated with linear polyacrylamide layers of different quality, was investigated by atomic force microscopy (AFM). After electropherograms were recorded of a synthetic mixture of proteins in a capillary having a given coating, the capillary was fragmented into many small pieces, and some of the fragments whose inner surface was easy to access by the cantilever were randomly selected and probed by AFM. A correlation between coating quality and capillary electrophoresis (CE) performance was observed. A comparison between both CE and AFM data from the same capillary indicates that those capillaries having zones with nonhomogeneous coatings, i.e., those having higher surface roughness, give poorer CE separation performances than those with a homogeneous polyacrylamide coating. The polymeric coating of these later capillaries showed typical thickness values of a few nanometers. The reduced CE performance observed in those capillaries with higher roughness is probably associated with the noxious adsorption of proteins directly onto the bare or thinly coated inner wall of the fused silica capillaries.

In capillary electrophoresis (CE), adsorption of proteins onto capillary walls seems to be the main reason for observed efficiency loss. ${ }^{1-3}$ Indeed, protein adsorption on the internal surface of the capillary is usually related to poor reproducibility in migration times and low protein recovery rates. ${ }^{4}$ Adsorption is most probably due to electrostatic interactions between positively

* Corresponding authors (address reprint request to diez-masa@fresno.csic.es or Alfredo_emilio.bruno@pharma.novartis.com).

(1) Hjertén, S. Electrophoresis 1990, 11, 659-690.

(2) Bushey, M. M.; Jorgenson, J. W. J. Chromatogr. 1989, 480, 301-310.

(3) Cifuentes, A.; Santos, J. M .; Frutos, M. de; Diez-M asa, J. C. J. Chromatogr., 1993, 652, 161-170.

(4) Towns, J. K.; Regnier, F. E. Anal. Chem. 1991, 63, 1126-1132. charged residues of the proteins and negatively charged silanol groups, which are intrinsic to the fused silica surface of uncoated capillaries. Thus, different methods have been developed to reduce the interaction between proteins and silica surfaces. Among them, capillaries with polymeric coatings have led to high efficiency and reproducible protein separations. ${ }^{3-13}$

An unsettled issue in CE is how thick and homogeneous the capillary coating should be in order to prevent protein adsorption effects. Two studies ${ }^{8,14}$ provided indirect information about the macroscopic properties and behavior of the capillaries. Towns and Regnier ${ }^{8}$ roughly estimated the thickness of a polyethyleneimine coating (a film bearing a high number of positive charges) using a modification of the picric acid assay for ion-pairing capacity. They claim that a thickness of about $3 \mathrm{~nm}$ suffices to mask the negative charges of the capillary wall and thus to preclude the adsorption of proteins. Malik et al., ${ }^{14}$ using gas chromatography measurements, concluded that a film thickness of $20 \mathrm{~nm}$ or higher of a polyethylene/ polypropyleneglycol coating (i.e., a medium polarity-stationary phase) strongly reduces electroosmotic flow and prevents protein adsorption. However, it has been argued ${ }^{15}$ that gas chromatographic determinations are of little use in characterizing polymeric coatings containing polar moieties. These studies ${ }^{8,14}$ did not address the effect of coating homogeneity

\footnotetext{
(5) Hjertén, S. J. Chromatogr. 1985, 347, 191-198.

(6) M cCormick, R. M. Anal. Chem. 1988, 60, 2322-2328.

(7) Bruin, G. J. M .; Chang, J. P.; Kulman, R. H.; Zegers, K.; Kraak, J. C.; Poppe, H. J. Chromatogr. 1989, 471, 429-436.

(8) Towns, J. K.; Regnier, F. E. J. Chromatogr. 1990, 516, 69-78.

(9) Smith, J. T.; El Rassi, Z. Electrophoresis 1993, 14, 396-406.

(10) Gilges, M.; Kleemiss, M. H.; Schomburg, G. Anal. Chem. 1994, 66, 20382046.

(11) Huang, M.; Vorkink, W. P.; Lee, M . L. J. Microcolumn Sep. 1992, 4, 233238.

(12) Nasabeh, W.; El Rassi, Z. J. Chromatogr. 1991, 559, 367-383.

(13) Erim, F. B.; Cifuentes, A.; Poppe, H.; Kraak, J. C. J. Chromatogr. 1995, 708, 356-361.

(14) Malik, A.; Zhao, Z.; Lee, M. L. J. Microcolumn Sep. 1993, 5, 119-125.

(15) Kohr, J.; Engelhardt, H. J. Microcolumn Sep. 1991, 3, 491-495.
} 
on the CE separation of proteins and thus become one leitmotiv in the present work.

Atomic force microscopy (AFM) has become an indispensable tool for characterizing surfaces presenting structures ranging from $100 \mu \mathrm{m}$ down, in some cases, to the atomic scale. ${ }^{16}$ Because AFM is hardly limited to any environment (e.g., ambient conditions, liquids, etc.) and offers the possibility to investigate both conducting and insulating surfaces, it is most suitable for probing the inner surface of capillaries. Indeed, Righetti's group demonstrated that AFM can be used to determine the roughness of a bare fused silica surface of a CE capillary ${ }^{17}$ and to prove the quality of polymeric coatings deposited onto oxidized silicon wafers. ${ }^{18,19}$ In their investigation, they noticed the remarkable smoothness of bare fused silica surfaces with a root-mean-squared roughness varying from 0.35 to $1.5 \mathrm{~nm}$ and showed that, in many cases, coatings do not provide a full coverage of the wafer surfaces which were used as a model system to mimic coated capillary surfaces. Furthermore, it has been shown ${ }^{20}$ that AFM can be used to probe the amount of proteins adsorbed to the capillary wall during migration, which is a key issue in controlling the efficiency in CE of proteins, as well as to image polyacrylamide-coated capillaries. ${ }^{21}$

Using AFM, we investigated polyacrylamide-coated capillaries. The main objective here is to characterize surface topographies, roughness, and morphology of differently coated capillaries previously used in the CE separation of proteins and to establish trends and correlations between surface quality and CE performance. The thickness of the polymeric coatings has also been determined directly from the depth of the holes observed in the coatings.

\section{EXPERIMENTAL SECTION}

CE Measurements. A P/ ACE 2000 HPCE (Beckman Instruments Inc., Fullerton, CA) electrophoresis apparatus controlled by a PS/ 2486 computer was used for CE experiments. Fused silica capillaries (Polymicro Technologies Inc., Phoenix, AZ) with inner and outer diameters of 50 and $360 \mu \mathrm{m}$, respectively, were used. Capillaries with a total length of $27 \mathrm{~cm}$ and an effective length of $20 \mathrm{~cm}$ were used for CE analysis. All injections were carried out by $\mathrm{N}_{2}$ pressure $(0.5 \mathrm{psi})$ at the anodic side for $1 \mathrm{~s}$. The detection wavelength was $214 \mathrm{~nm}$. All the data were collected and analyzed using commercial software (System Gold, Beckman).

Each coated capillary (see below) was first installed in the CE instrument and filled with $50 \mathrm{mM}$ acetic acid/sodium acetate buffer at $\mathrm{pH}$ 5. A solution of basic proteins in water $(0.5 \mathrm{mg} / \mathrm{mL}$ of lysozyme, $0.6 \mathrm{mg} / \mathrm{mL}$ of ribonuclease $A$, and $0.4 \mathrm{mg} / \mathrm{mL}$ of $\alpha$-chymotrypsinogen) was injected into the capillary and run at a voltage of $12 \mathrm{kV}$. After five injections, each capillary was washed with $\sim 20$ volumes of M illi-Q water (M illipore, Bedford, M A) and emptied by flushing dried $\mathrm{N}_{2}$ (5 psi) for $2 \mathrm{~min}$. Finally, the

(16) Hansma, P. K.; Elings, V. B.; M arti, O.; Bracker, C. E. Science 1988, 242, 209-213.

(17) Barberi, R.; Giocondo, M .; Bartolino, R.; Righetti, P. G. Electrophoresis 1995, 16, 1445-1450.

(18) Barberi, R.; Bonvent, J. J.; Bartolino, R.; Roeraade, J.; Capelli, L.; Righetti, P. G. J. Chromatogr. B 1996, 683, 3-13.

(19) Capelli, L.; Ermakov, S. V.; Righetti, P. G. J. Biochem. Biophys. M ethods 1996, $32,109-124$.

(20) Bonvent, J. J.; Barberi, R.; Bartolino, R.; Capelli, L.; Righetti, P. G J. Chromatogr. A 1996, 756, 233-243.

(21) Kaupp, S.; Waetzig, H. J. Chromatogr. A 1997, 781, 55-65. capillaries were detached from the CE apparatus and prepared for the AFM study.

AFM Measurements. In AFM, a flexible cantilever with an integrated tip (tip radius of $\sim 10 \mathrm{~nm}$ ) raster scans the sample surface, giving access to topographical information with nanometer resolution. ${ }^{22}$ This sharp force sensor bends as the tip is brought closer or in contact with the sample surface according to attractive or repulsive interaction forces. A laser beam focused on the back of the cantilever, together with a position-sensitive photodetector, measures minute sensor deflections. Upon controlling the sensor deflection and thus the interaction between the tip and a surface by an electronic feedback system, the motion of the sensor tip is recorded as a function of tip position. This allows the reconstruction and visualization of the surface topography. The AFM measurements were carried out with a commercial instrument (NanoScope III multimode, Digital Instruments, Santa Barbara, CA) under ambient conditions. All reported images were acquired in the AFM tapping mode with silicon cantilevers (Nanoprobe, Wetzlar, Germany).

Prior to being probed by AFM , the capillaries were fragmented with the help of a compression stress relaxometer ( $\mathrm{M} \mathrm{K} \mathrm{II,} \mathrm{Wallace,}$ Croydon, England). Selected fragments, exposing their inner surface to the AFM cantilever, were selected under an optical microscope and mounted on the AFM sample holder with a double-sided sticky tape. Several samples, representative of different sections of the capillary, were studied in order to obtain a representative picture of the capillary being characterized.

Reagents. Lysozyme (chicken egg white), ribonuclease A (bovine pancreas), and $\alpha$-chymotrypsinogen (bovine pancreas) were purchased from Sigma Chemical Co. (St. Louis, MO) and used as received. The proteins were dissolved in M illi-Q water at concentrations as individually stated. All samples were stored at $-4{ }^{\circ} \mathrm{C}$ and warmed to room temperature before use. Acetic acid (Merck, Darmstadt, Germany) was used in the running buffer. The $\mathrm{pH}$ of this solution was adjusted using $1 \mathrm{M}$ sodium hydroxide.

(7-0 ct-1-enyl)trimethoxysilane (ABCR, Karlsruhe, Germany), acrylamide (caution: neurotoxic product, special precautions should be taken for handling it), ammonium persulfate, and TEMED (all from Schwarz, Cleveland, $\mathrm{OH}$ ) were used for the preparation of the coated capillaries.

Polyacrylamide Coating P rocedures. Used capillaries were coated with linear polyacrylamide (PAA) according to (except for some modifications mentioned below) a published protocol. ${ }^{5}$ Capillaries were first rinsed with $1 \mathrm{M} \mathrm{NaOH}(2 \mathrm{~h}, 5 \mathrm{~mL})$, washed with water $(1 \mathrm{~h}, 2.5 \mathrm{~mL})$, and purged with $\mathrm{N}_{2}$ gas at $235^{\circ} \mathrm{C}$ for 2 h. A solution of $20 \mu \mathrm{L}$ of acetic acid, $20 \mu \mathrm{L}$ of (7-oct-1-enyl)trimethoxysilane, and $4 \mathrm{~mL}$ of methanol was "pressed" through the capillaries with nitrogen gas for $2 \mathrm{~h}$ at room temperature. Afterward, the capillary was rinsed with methanol ( $1 \mathrm{~h}, 2.5 \mathrm{~mL})$. Once the bifunctional agent had reacted with the capillary wall, Hjertén's procedure ${ }^{5}$ was employed in order to attach the linear PAA.

\section{RESULTS AND DISCUSSION}

Analysis of the recorded AFM images of coated capillaries gave information about two aspects relevant to $C E$ : homogeneity and

(22) Sarid, D. Scanning Force M icroscopy; Oxford University Press: New York, 1991. 
thickness of the coating. To correlate this information with CE performance, a first set of experiments was designed to assess the separation efficiency of identical basic protein mixtures in capillaries having different quality coatings. The buffer composition and $\mathrm{pH}$ were chosen in such a way that interactions between proteins and capillary wall were enhanced. This interaction has been assumed ${ }^{3,8}$ to take place in the uncoated or thinly coated parts of the capillary; the present AFM data support this assumption. The same images also contain information about the thickness of the linear polyacrylamide coating.

Figure la shows a CE separation of the mixture of basic proteins obtained with a capillary (referred to as capillary 1 hereafter) coated with linear PAA. The typical AFM image of a $5 \times 5 \mu \mathrm{m}^{2}$ area of the inner wall surface of capillary 1 is shown in Figure $1 \mathrm{~b}$ at a vertical $z$-scale of $40 \mathrm{~nm}$. To better visualize the surface morphology, the vertical z-scale of the inset was chosen to be $5 \mathrm{~nm}$. The separation efficiency (Figure 1a) is relatively high for these types of systems (i.e., higher than 1 million plates/ $m$ as determined from $\alpha$-chymotrypsinogen, peak 3 ), while peak tailing is hardly noticeable, indicating that the proteincapillary wall did not degrade performance. As indicated in Figure 1 , capillary 1 is uniformly coated, showing a smooth and homogeneous surface with only a few small holes with diameters of $\sim 10 \mathrm{~nm}$ and some protrusions smaller than $5 \mathrm{~nm}$. The depth of the small holes, representing a lower limit of the polymer thickness and possibly noncoated zones, ${ }^{18,19}$ was estimated to be 2-3 $\mathrm{nm}$. Because the coated area is rather homogeneous, the depth of the holes should closely correspond to the film thickness. AFM images from different capillary pieces, randomly taken from the same column, showed topography and film thickness similar to those presented above, indicating a good homogeneity of the coating along the column. Furthermore, it can also be inferred from the same data, as well as from the results published by Righetti et al., ${ }^{17}$ than the roughness of the coatings does not correlate with the quality of the fused silica substrate, which is extremely flat, having a RMS roughness of $0.4-0.5 \mathrm{~nm}$ (see Table 1).

To gain more insight into the interrelation between the topography of the capillary coatings and its CE behavior, we studied a second capillary, capillary 2, which appears to be poorly coated with linear polyacrylamide (as compared with that shown in Figure 1b). The defective behavior of this column was suspected prior to any experiment because during its preparation almost no gelification of the coating solution was observed, probably due to the well-known sensitivity of this reaction to oxygen, which acts as an inhibitor of persulfate-driven polymerizations. ${ }^{23}$ Thus, using this column, the different CE performance of capillaries with chemically identical coatings related to their different coating topography could be investigated. Figure 2 shows a CE separation of the test proteins in this poorly coated linear PAA capillary (Figure 2a), together with a $5 \times 5 \mu \mathrm{m}^{2}$ AFM image of its inner surface at a vertical z-scale of $40 \mathrm{~nm}$ (Figure $2 b)$. The protein peaks in this case display large tailings, suggesting strong interactions between proteins and capillary wall. For lysozyme (the most positively charged of the three proteins injected, peak 1 in Figure 2a), adsorption is so strong that it can

(23) Righetti, P. G.; Bossi, A.; Giglio, M .; Vailati, A.; Lyubimova, T.; Briskman, V. A. Electrophoresis 1994, 15, 1005-1013. (a)
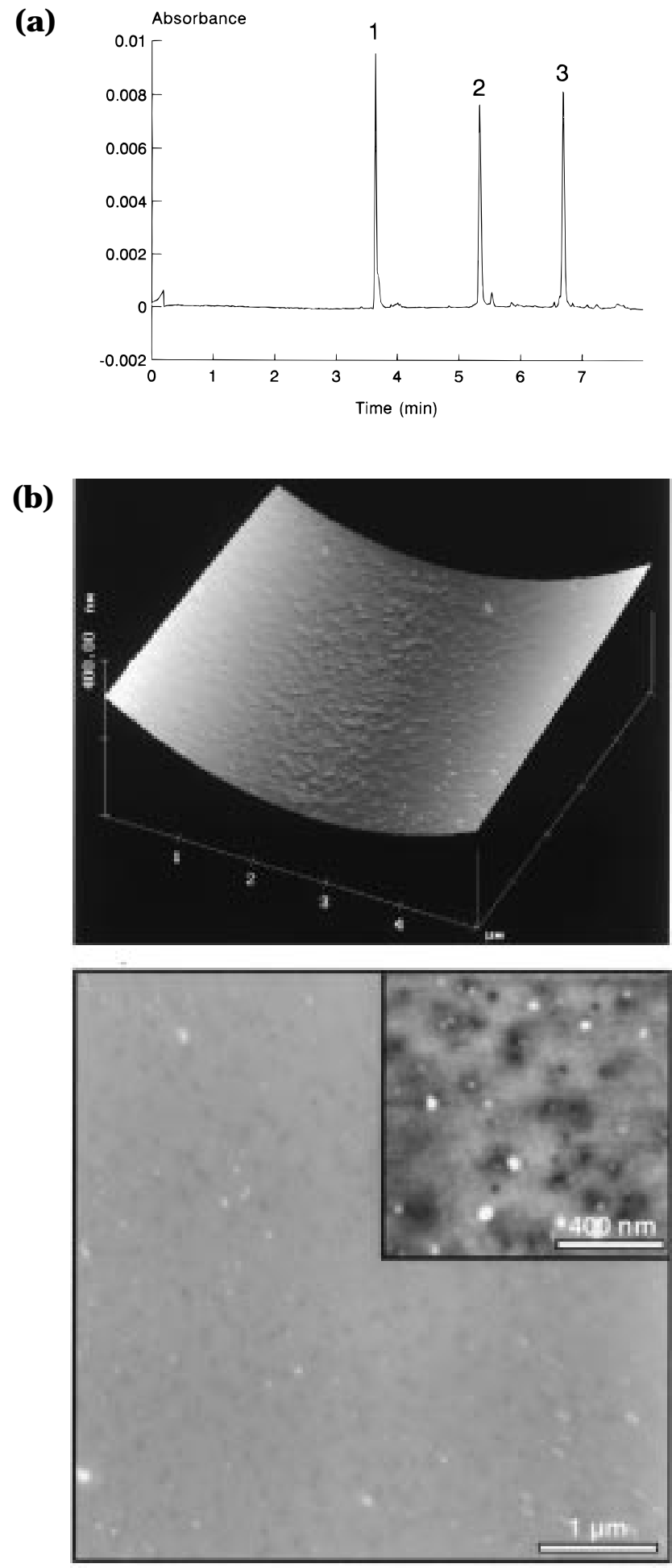

Figure 1. (a) Electropherogram of a mixture of three basic proteins obtained with linear polyacrylamide-coated capillary (referred to as capillary 1 in the text). Separation conditions: capillary i.d., $50 \mu \mathrm{m}$; total length, $27 \mathrm{~cm}$; effective length, $20 \mathrm{~cm}$; running buffer, $50 \mathrm{mM}$ acetic/acetate, $\mathrm{pH} 5$; hydrodynamic injection, 0.5 psi during $1 \mathrm{~s}$; running voltage, $12 \mathrm{kV}$. The sample mixture (peak assignments are given in brackets) contains lysozyme (1), ribonuclease A (2), and $\alpha$-chymotrypsinogen (3). Detection absorption at $\lambda=214 \mathrm{~nm}$. (b) Typical AFM images $\left(5 \times 5 \mu \mathrm{m}^{2}\right)$ of a surface area shown in 3D representation (top) and color-encoded topview representation (bottom and inset). In both topview images, the capillary curvature has been removed for better surface visualization, and the vertical image $z$-scale was set to 40 and $5 \mathrm{~nm}$ (inset). 
(a)

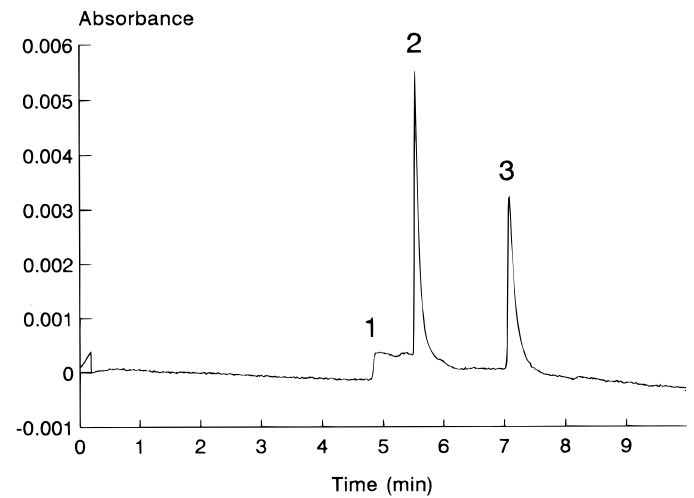

(b)
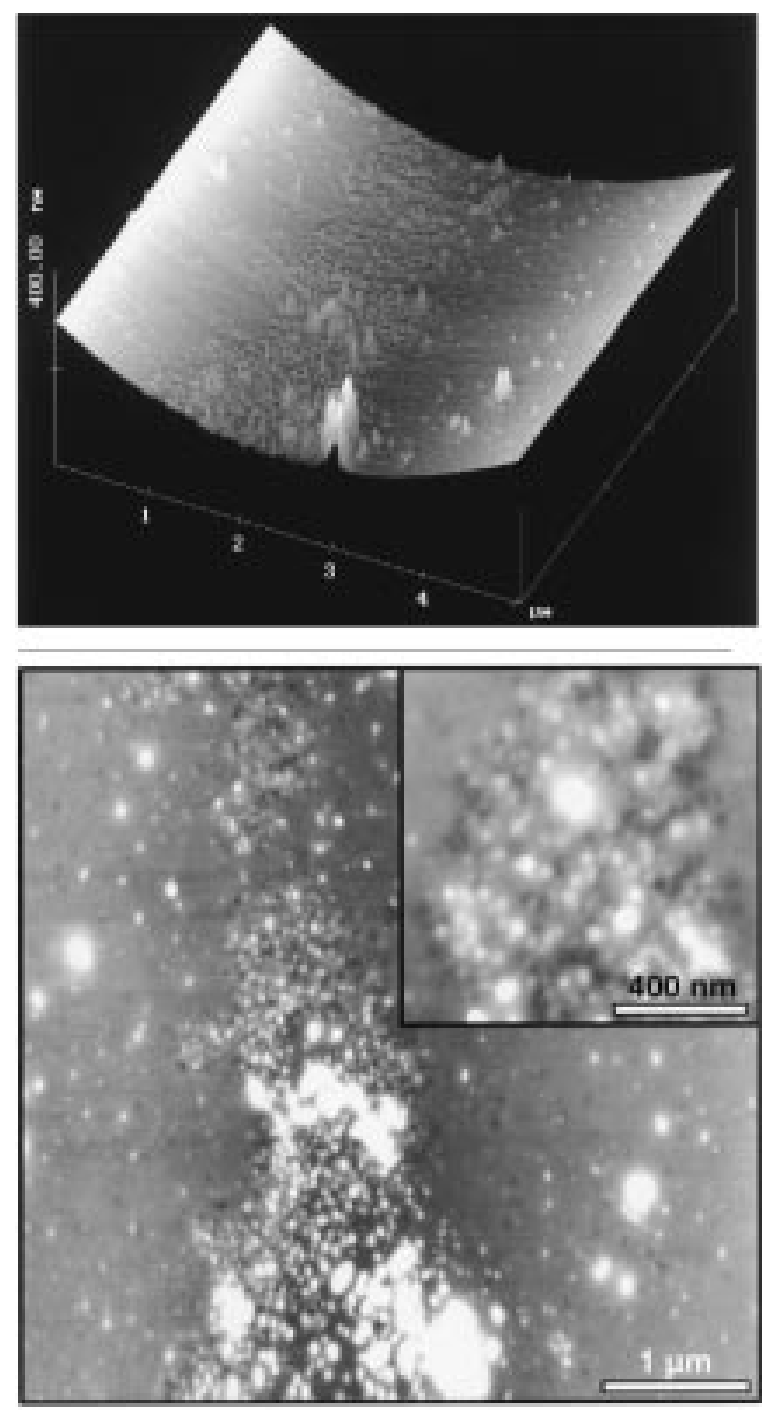

Figure 2. (a) Electropherogram of a mixture of three basic proteins obtained with a capillary poorly coated with linear polyacrylamide (referred to as capillary 2 in the text). Separation conditions are as given in Figure 1. (b) Typical AFM images $\left(5 \times 5 \mu \mathrm{m}^{2}\right)$ of a surface area shown in 3D representation (top) and color-encoded topview representation (bottom and inset). In both topview images, the capillary curvature has been removed for better surface visualization, and the vertical image $z$-scale was set to $40 \mathrm{~nm}$.

hardly be detected. As can be seen in Figure $2 \mathrm{~b}$, the topography of this coating displays a large heterogeneity, showing numerous pinholes and hillocks, which in some cases protrude more than
Table 1. AFM and CE Data for Bare Fused Silica and Capillaries 1 and 2 (See Text)

\begin{tabular}{|c|c|c|c|}
\hline capillary & $\begin{array}{c}\text { RMS } \\
\text { roughness }^{\mathrm{a}}(\mathrm{nm})\end{array}$ & $\begin{array}{c}\mathrm{PP} \\
\text { roughness }^{\mathrm{a}}(\mathrm{nm})\end{array}$ & $\begin{array}{c}\mathrm{N}_{\max } \\
\text { (plates/m) }\end{array}$ \\
\hline bare silica & $0.4-0.5$ & $1-2$ & \\
\hline $\begin{array}{l}\text { capillary } 1 \\
\text { capillary } 2\end{array}$ & $0.7-1.2$ & $10-20$ & $1.1 \times 10^{6}$ \\
\hline capillary 2 & $1.6-7.5$ & $30-40$ & $0.2 \times 10^{6}$ \\
\hline
\end{tabular}

a RMS = root-mean-square; PP = peak-to-peak. All roughness numbers were determined after correcting for the intrinsic capillary curvature.

$40 \mathrm{~nm}$ above the surface, contrasting the features in Figure $1 \mathrm{~b}$, which are displayed at an identical vertical scale. These protrusions can be associated with agglomerations of PAA induced by local polymerization of the coating solution. Similar topography was also observed in other fragments of the same capillary randomly chosen. However, in other sections of the same capillary, the topography observed was similar to that of capillary 1 (Figure $1 b$ ), indicating that uncoated and/ or thinly coated regions of capillary alternate with others, presenting a much more homogeneous coating. No regions displaying topographies such as that shown in Figure $2 b$ were observed in capillary 1.

Therefore, we assume that the poor CE performance delivered by capillary 2 is due to the large inhomogeneity of its coating. This heterogeneity of the coating, with its small noncoated areas, can induce local fluctuations in the electroosmotic flow, degrading the electrophoretic band profile, manifesting in a decrease in the resulting CE performance. ${ }^{24}$ Similar results could be observed on the regions coated with a very thin layer of polymer, which are known ${ }^{8}$ to be rather inefficient in preventing protein adsorption. The topography observed for the coating in different sections of this capillary, together with the CE separation of basic proteins, suggests that capillaries presenting bad CE properties could have some homogeneously coated segments alternating with others, presenting large inhomogeneities.

CE and AFM relevant data are summarized in Table 1, which also includes AFM data from bare silica capillary for comparative purposes (data not shown). The AFM values of RMS (i.e., representing the averaged root-mean-square roughness) and PP (peak-to-peak) roughness were obtained from four different zones of each capillary and calculated upon correcting for the intrinsic capillary curvature. The $\mathrm{N}_{\max }$ column in Table 1 refers to the maximum efficiency values obtained for $\mathrm{CE}$ analysis of the basic proteins as reported above, and $A_{s}$ refers to the asymmetry factor ${ }^{25}$ for these proteins. As can be seen, the roughness values are much higher for the two coated capillaries than for the bare silica. This is probably due to the intrinsic polymer morphology grafted on the capillary wall increasing the surface roughness. More interestingly, a correlation between the roughness values, the homogeneity of the surface (as seen in Figures $1 b$ and $2 b$ ), and the CE performance for coated capillaries (efficiency and asymmetry values) can be deduced. Hence, the lower the roughness value and the more homogeneous the surface of the inner wall of a capillary, the higher is its CE performance.

(24) Towns, J. K.; Regnier, F. E. Anal. Chem. 1992, 64, 2473-2478.

(25) Snyder, L. R., Kirkland, J. J. Introduction to M odern Liquid Chromatography, John Wiley and Sons: New York, 1979, Chapter 5. 


\section{CONCLUSIONS}

The presented results show conclusive evidence for a correlation between the observed CE performance with the surface topography of capillary coatings. A homogeneous film thickness of a few nanometers along the entire capillary is necessary to reduce the adsorption of basic proteins on the capillary wall. The existence of poorly coated areas dramatically decreases CE performance because adsorption occurs on the uncoated and perhaps even on thinly coated areas of poorly coated capillaries. We envision that the ability of AFM to probe surfaces on a molecular scale will play an important role in the understanding and development of new capillary coatings for CE.

\section{ACKNOWLEDGMENT}

A.C. and J.C.D.-M . thank the Commission of the European Community (Training and M obility of Researchers, Contract $\mathrm{No}$. ERBFM BICT 950003), and D GICYT of Spain (Project N o. M AT960564) for financial support. J.F. and D.A. acknowledge discussions and support from the University of Basel (H.-J. Güntherodt).

Received for review February 9, 1998. Accepted May 19, 1998. 